\title{
Novel approach to the characterization of melanoma associated-peptide-specific CTL lines from Japanese metastatic melanoma patients
}

\author{
YASUTO AKIYAMA ${ }^{1}$, KOUJI MARUYAMA ${ }^{1}$, SACHIKO TAI $^{1}$, MASAKO TAKIKAWA ${ }^{1}$, CHIE OHSHITA $^{1}$, \\ AKIFUMI YAMAMOTO $^{4}$, NAOYA YAMAZAKI ${ }^{3}$, YOSHIO KIYOHARA ${ }^{2}$ and KEN YAMAGUCHI $^{1}$ \\ ${ }^{1}$ Immunotherapy Division, Shizuoka Cancer Center Research Institute, ${ }^{2}$ Department of Dermatology, Shizuoka Cancer \\ Center Hospital, 1007 Shimonagakubo, Nagaizumi-cho, Sunto-gun, Shizuoka 411-8777; ${ }^{3}$ Department of Dermatology, \\ National Cancer Center Hospital, 5-1-1 Tsukiji, Chuo-ku, Tokyo 104; ${ }^{4}$ Department of Dermatology, \\ Saitama Medical University, 38 Moroyama, Iruma-gun, Saitama 350-0495, Japan
}

Received March 26, 2008; Accepted May 21, 2008

DOI: 10.3892/ijo_00000025

\begin{abstract}
Melanoma-associated antigens, MART-1, tyrosinase, gp100 and MAGEs, are typical melanoma-specific tumor antigens which can potently induce immune responses in metastatic melanoma patients treated with peptide vaccines. In the present study, we established a dendritic cell (DC)based HLA-A2 melanoma-associated peptide (MART-1 or gp 100)-specific CTL induction method and characterized the CTLs using HLA-A2 tetramer staining in 6 cases of HLA$\mathrm{A}^{+}$melanoma treated with DC vaccines. Peripheral blood mononuclear cells (PBMC) from patients were stimulated twice with MART-1 A2 peptide-pulsed DCs in the presence of a low dose of IL-2. To boost CTL populations, CTL lines were further stimulated twice with MART-1 A2 peptidepulsed T2 cells. The frequency of MART-1 A2 tetramerpositive CTLs increased from $0.16 \%$ (prior to stimulation) to $2.15 \%$ (after DC stimulation), and reached $46.5 \%$ on average (after additional T2 stimulation) in 4 cases which showed a successful expansion. The absolute numbers of MART-1 A2 tetramer-positive CTLs increased from 187- to 619-fold (average, 415-fold) compared to prior to DC stimulation. CTL assays using MART-1-specific CTL lines demonstrated potent killing activity against MART-1 peptide-pulsed T2 cells or HLA-A2+ melanoma cell lines in accordance with the frequency of tetramer-positive CTLs. Finally, we were successful in identifying melanoma peptide-specific T-cell receptor (TCR) cDNAs in 2 cases for MART-1 and 1 case for gp100 using the anti-TCR MoAb-based sorting as a novel
\end{abstract}

Correspondence to: Dr Yasuto Akiyama, Immunotherapy Division, Shizuoka Cancer Center Research Institute, 1007 Shimonagakubo, Nagaizumi-cho, Sunto-gun, Shizuoka 411-8777, Japan

E-mail: y.akiyama@scchr.jp

Key words: DC vaccine, metastatic melanoma, melanomaassociated HLA-A2 peptide, tetramer sorting, electroporation approach instead of a conventional cell cloning, and confirmed peptide-specific IFN- $\gamma$ production in TCR cDNA-transduced naïve $\mathrm{T}$ cells. The results showed that cloned TCR cDNAs were efficient in reconstituting tumor-specific cytotoxicity and good candidates for novel immunotherapy.

\section{Introduction}

Melanoma-associated antigens are categorized as class I human leukocyte antigen (HLA)-restricted cancer/testis antigens (1) which are considered to be immunogenic to the immune system because they are hardly expressed in normal tissues except testis. However, malignant melanoma is the most well known cancer in which multiple tumor-specific antigens have been defined and utilized in vaccination strategies as peptide vaccines or peptide-pulsed DC vaccines $(2,3)$. Our group has been running a clinical phase I/II trial of peptide cocktail-pulsed DC vaccines in metastatic melanoma patients for some years. We reported that almost all cases showed more than 2 peptide-specific CTL responses in blood and 2 cases had clinical responses (1 CR, 1 PR) (4).

Few studies have focused on the characterization or determination of peptide-oriented single specific CTL clones from melanoma patients treated with DC vaccines. Recently, specific CTLs or tumor-infiltrating lymphocytes (TILs) have been successfully cloned from blood or tumors of melanoma patients (5-8). In some cases, MART-1 or gp100-specific CTL clones obtained from the tumor tissue were expanded, and could be utilized for adoptive immunotherapy $(7,8)$. As to other types of cancers, a very small number of TILs were expanded to isolate tumor-specific clones from a bulk of TILs and utilized to search for novel tumor antigens in a tumor-derived complementary DNA library $(9,10)$. However, cloning from a bulk of CTLs is time-consuming and usually costly.

In the present study, we established an easy and efficient method for the expansion and separation of a very small number of melanoma peptide-specific CTLs using HLA-A2 peptide tetramer or TCR-specific MoAb-based cell sorting. 
Through the molecular cloning of melanoma peptide (MART-1 or gp100)-specific TCRs, the biological characterization of each CTL line was performed in Japanese metastatic melanoma patients given DC vaccines.

\section{Materials and methods}

Reagents and cell lines. Recombinant human (rh) granulocyte macrophage colony-stimulating factor (GM-CSF), rh-interleukin (IL)-2, rhIL-4, rhIL-7, and TNF- $\alpha$ were purchased from PeproTech Inc. (Rocky Hill, NJ). GM-CSF and IL-4 were used at $50 \mathrm{ng} / \mathrm{ml}$ for dendritic cell (DC) cultures. Mouse monoclonal antibodies (MoAbs) to human CD1a, CD3, CD8, CD11c, HLA-ABC (class I), HLA-DR (class II), CD80, CD83, CD86 and FITC as well as PE-labeled anti-human IFN- $\gamma$ antibody were all obtained from PharMingen (San Diego, CA, USA). HLA*0201 MART-1 (ELAGIGILTV), gp100 and Influenza matrix protein (Flu-MP) tetramers were purchased from MBL (Nagoya, Japan). TCR Vß repertoire kit and FITC-labeled anti-specific TCRVß repertoire MoAbs were from Beckman Coulter Inc. (CA, USA). The T2 cell line was purchased from the American Type Culture Collection (Manassas, VA, USA). Human melanoma cell lines, C32 and RPMI7951, were obtained from ATCC and NCC-KT was provided by Dr Y. Hamanaka (Yamaguchi University School of Medicine, Ube, Japan).

Synthetic peptides. The sequences of melanoma-associated peptides used in the present study are MART-1 ${ }_{27-35}$ (AAGIGILTV), gp100 $209-217$ (IMDQVPFSV) and Flu-MP $58-66$ (GILGFVFTL). These peptides were synthesized as reported previously (11).

CTL induction cultures. PBMCs from 6 cases of HLA$\mathrm{A}^{*} 0201^{+}$metastatic melanoma were used for in vitro $\mathrm{CTL}$ inductions. The clinical research using PBMC from melanoma patients was approved by the Institutional Review Board of Shizuoka Cancer Center, Shizuoka, Japan. All patients gave written informed consent. All cases of metastatic melanoma were given HLA-A2 melanoma-associated peptide-pulsed DC vaccines in clinical trial reported previously (4). Briefly, after non-adherent PBMCs were stimulated twice with MART-1 A2 peptide-pulsed mature DCs (most cells positively stained with CD83 MoAb), cells were boosted in RPMI-1640 medium containing L-glutamine (2 mM), penicillin $(100 \mathrm{U} / \mathrm{ml})$, streptomycin $(100 \mathrm{U} / \mathrm{ml})$ and $5 \% \mathrm{AB}$ human serum referred to as CTL medium with 2 rounds of stimulation with MART-1 peptide-pulsed T2 cells. In case 3, gp100 peptide was also used for the induction. Finally, expanded peptide-specific CTLs were utilized for various experiments or cell sorting.

$T C R$ repertoire staining with anti-TCR MoAb panel. The staining profile of CTLs during the expansion procedure was monitored using a TCR Vß repertoire kit, and after DC plus T2 cell-based expansion, major populations positively stained with the specific anti-TCR antibody were determined.

Tetramer staining. Cultured CTLs were stained with both FITC-anti-CD8 MoAb and PE-labeled HLA-A2 MART-1,
Table I. Analysis of peptide-specific CTL production from HLA-A*0201-positive melanoma patients.

\begin{tabular}{lccccccc}
\hline \multirow{2}{*}{$\begin{array}{l}\text { Case } \\
\text { no. }\end{array}$} & \multicolumn{3}{c}{ Total cell no. $\left(\times 10^{7}\right)$} & & \multicolumn{3}{c}{ HLA-A2 tetramer $(\%)$} \\
\cline { 7 - 8 } \cline { 6 - 8 } & Pre & 2DC & 2DC+2T2 & & Pre & 2DC & 2DC+2T2 \\
\hline 1 & 2.4 & 2.5 & 2.0 & & 0.14 & 0.89 & 31.7 \\
2 & 2.0 & 3.6 & 3.9 & & 0.35 & 4.64 & 78.8 \\
3 & 2.0 & 2.2 & 4.8 & & 0.12 & 1.73 & 40.5 \\
$3^{*}$ & 2.0 & 9.3 & - & & 0.13 & 44.3 & - \\
4 & 2.0 & 1.4 & 0.62 & 0.02 & 1.32 & 35.0 \\
5 & 2.0 & 1.2 & - & 0.05 & 0.12 & N.D. \\
6 & 1.7 & 0.48 & - & 0.03 & 0.11 & N.D.
\end{tabular}

Pre, before starting CTL induction; 2DC, after 2 rounds of peptidepulsed DC stimulation; 2DC+2T2, after 2 rounds of peptide-pulsed T2 stimulation in addition to 2DC; N.D., not detected. Each value shows the mean for 2 experiments. " gp100 A2 peptide.

gp100, or Flu-MP tetramer as previously described (12). Cells were analyzed on a flow cytometer.

CTL killing assay. CTL killing was assessed with the DELFIA non-radioactive cytotoxicity assay (Perkin-Elmer Inc., Waltham, MA, USA). Briefly, target cells (T2 cells, melanoma cell lines) were labeled with a fluorescence enhancing ligand, BATDA, and mixed with effector cells at an E/T ratio of 10 for $4 \mathrm{~h}$. The supernatant collected from the CTL culture was incubated with europium solution, and the EuTDA level was measured in real-time on a fluorometer, ARVOsx-2 (Perkin-Elmer Inc.).

Intracellular IFN- $\gamma$ staining. CTLs from 4 melanoma cases were pre-incubated with MART-1 peptide-pulsed or un-pulsed $\mathrm{T} 2$ cells for $4 \mathrm{~h}$, and stained intracellularly with anti-human IFN- $\gamma$ MoAb, HLA-A2 MART-1 peptide-specific tetramer, and/or anti-specific TCR MoAb. The stained cells were analyzed on a flow cytometer.

CTL sorting by MACS. HLA-A2 MART-1 and gp100 peptide tetramer-based or TCR MoAb-based CTL sorting was performed using the autoMACS (magnetic cell sorting) system (Miltenyi, Germany). Briefly, we used a specific PE-labeled tetramer or FITC-labeled TCR-specific MoAb as primary antibody, and anti-PE or FITC MoAb microbeads as secondary antibody. The purity of the tetramer ${ }^{+}$or specific $\mathrm{TCR}^{+}$CTLs was $>98 \%$ (data not shown). Purified CTLs were sequentially used for PCR cloning of the TCR gene.

T2-stimulated IFN- $\gamma$ production from peptide-specific CTL line sorted by TCR-specific MoAb. Expanded CTL lines from case 2 were sorted by FITC-labeled anti-TCR BV28 or anti-TCRBV12 MoAb using the autoMACS. After an overnight incubation with CTL medium, sorted CTLs $\left(1 \times 10^{5}\right)$ and peptide-pulsed T2 cells $\left(1 \times 10^{5}\right)$ were co-incubated in a round bottomed-96-well microculture plate for $24 \mathrm{~h}$. Finally, 

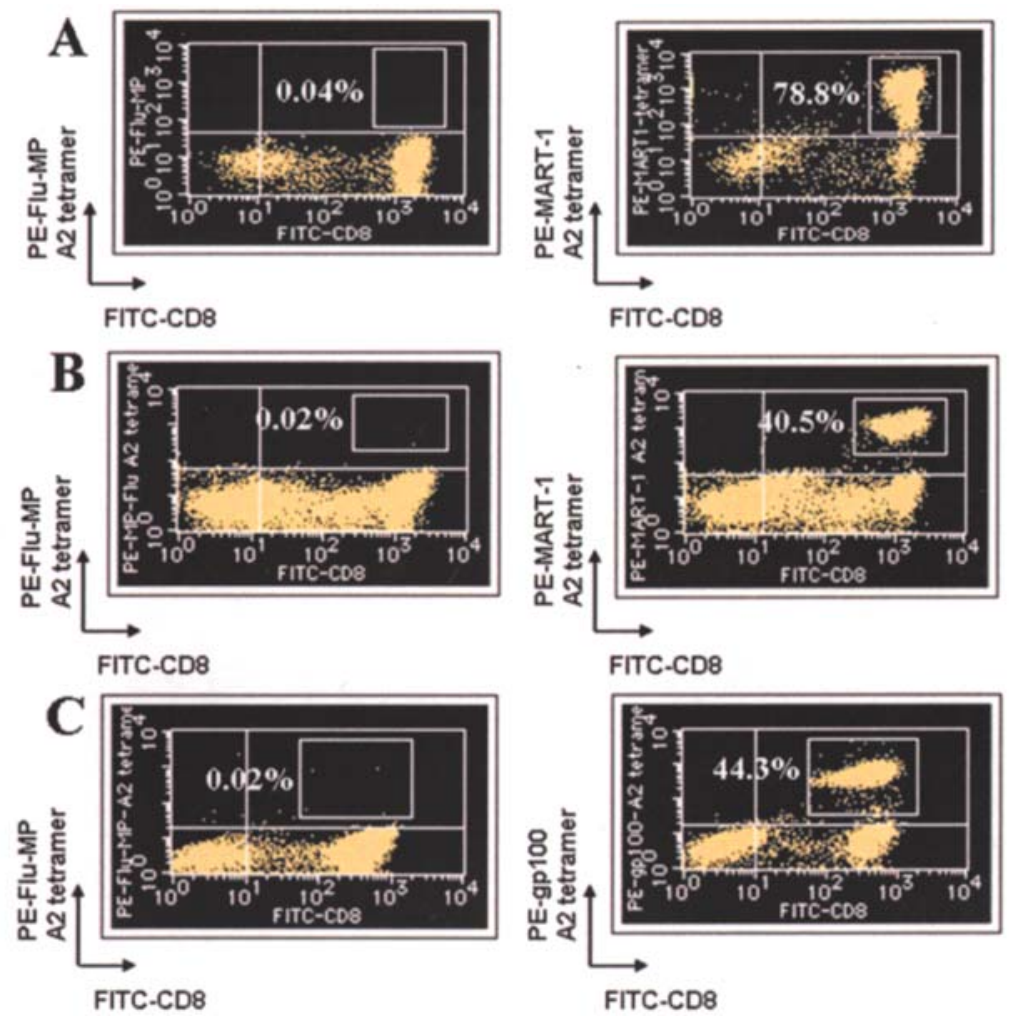

Figure 1. Expansion of HLA-A2 peptide tetramer-positive CTLs after stimulation 4 times with peptide-pulsed DCs and T2 cells. MART-1 A2 peptide-specific CTL lines from case 2 (A) and case 3 (B). Gp100 A2 peptide-specific CTLs from case 3 (C). The left side of the panel shows the staining of influenza (Flu)-MP A2 peptide tetramer, and the right side, the staining of MART-1 or gp100 A2 peptide tetramer.

supernatants were collected and IFN- $\gamma$ levels were measured using an ELISA kit specific for human IFN- $\gamma$ (Biosource, Camarillo, CA, USA).

PCR cloning and sequencing of MART-1 or gp100 peptidespecific TCRBV cDNA. Total RNA of sorted CTLs was prepared with a kit, Nucleospin RNA II (Machery-Nagel, Germany), and aliquots of $2 \mu \mathrm{g}$ were subjected to reverse transcription using oligo (dT) primer and SuperScript II (Invitrogen, CA, USA). The first strand cDNA was amplified by PCR using KOD Polymerase (Toyobo, Japan) according to the manufacturer's instructions and coding region-specific primers for TCRBV28 and TCRBC1 (MART-1 peptidespecific TCR), or TCRBV12-4 and TCRBC2 (gp100 peptidespecific TCR). The primer sequences are as follows: 5'-GC AGCCATGGGAATCAGGCTCCTCTGT-3' for TCRBV28, 5'-TCAGAAATCCTTTCTCTTGACCATGGC-3' for TCRBC1, 5'-TCTGCCATGGACTCCTGGACCCTCTGC-3' for TCRBV12-4, and 5'-CTAGCCTCTGGAATCCTTTCTC TTGAC-3' for TRBC2. The PCR product was separated by electrophoresis on a $1.5 \%$ agarose gel, and the band of appropriate size (bp) was excised and extracted from the gel. The recovered DNA fragment was cloned into the plasmid pCR-Blunt (Invitrogen), and its sequence was determined using BigDye Terminator reagent and a 3130xl Genetic Analyzer (Applied Biosystems, CA, USA). To confirm DNA sequences, $>12$ independent clones were analyzed for each TCR gene. The confirmed cDNA sequences for each TCR gene were analyzed by a WEB tool of IMGT (JunctionAnalysis, http://imgt.cines.fr/).
Construction of expression plasmid. The MART-1 peptidespecific TCR gene cloned in pCR-Blunt was digested with PstI and blunted with T4 DNA polymerase (Takara, Japan), and then digested with BamHI. The resultant DNA fragment was cloned into the blunted NheI-BglII site of a pmax expression plasmid (Amaxa, Cologne, Germany). The EcoRV-BamHI fragment of the gp100 peptide-specific TCR gene from pCR-Blunt was cloned into pmax as in the case of the former TCR gene fragment.

TCRBV gene transduction into primary naive $T$ cells. The plasmid vector pmax was utilized for making the construct containing GFP, cloned specific TCR genes, or vehicle. A $\mathrm{T}$ cell transfection kit (Nucleofector ${ }^{\mathrm{TM}}$, Amaxa) and a Nucleofector device (Amaxa) were used according to the manufacturer's instructions. Prior to electroporation, all lymphocytes including $\mathrm{T}$ cells were usually stimulated with anti-CD3 $(2 \mu \mathrm{g} / \mathrm{ml})$ and CD28 MoAb $(1 \mu \mathrm{g} / \mathrm{ml})$ for 5 days in GT-T503 medium and collected for the gene transduction procedure. The expression of TCR protein was analyzed on a flow cytometer using anti-TCRBV9 and BV28 (in MART-1) or anti-TCRBV12 (in gp100) MoAb.

IFN- $\gamma$ production by specific TCR gene-transduced naïve $T$ cells. Two days after electroporation, naïve $\mathrm{T}$ cells transduced with mock, GFP, or a specific TCR gene were harvested and incubated with melanoma peptide-pulsed $\mathrm{T} 2$ cells or the melanoma cell line for $24 \mathrm{~h}$. The supernatant was collected and the IFN- $\gamma$ level was measured using an ELISA kit specific for human IFN- $\gamma$. 


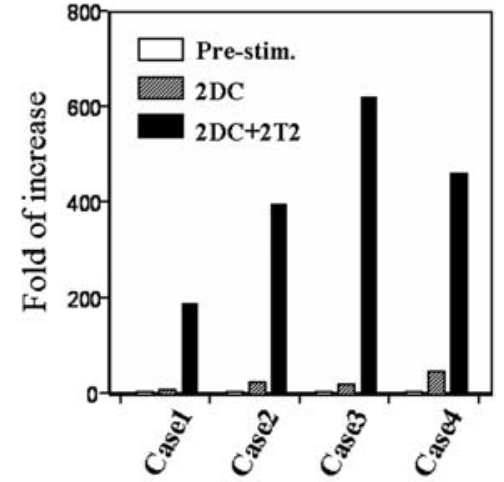

Figure 2. Expansion of MART-1 A2 peptide tetramer-positive CTLs after 4 rounds of stimulation. PBLs from 4 HLA-A2 $2^{+}$melanoma patients were stimulated ex vivo up to 4 times. Each column shows the mean value for results from 2 experiments. Pre-stim, pre-stimulation; 2DC, 2 rounds of DC stimulation; 2DC+2T2, 2 rounds of stimulation by DCs followed by 2 rounds of stimulation by $\mathrm{T} 2$ cells.
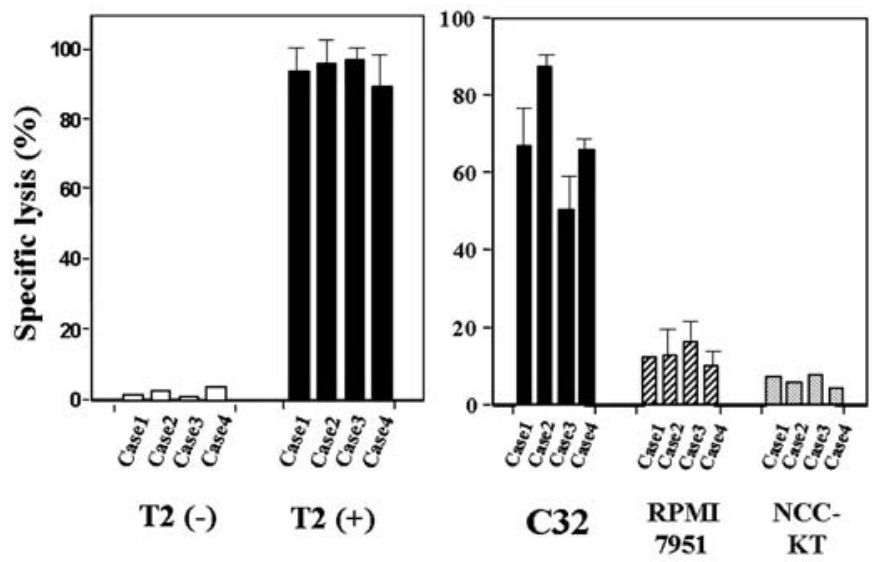

Figure 3. Cytotoxic activity of expanded MART-1 A2 peptide-specific CTL lines from 4 HLA-A2 ${ }^{+}$melanoma patients. Target cells were labeled with fluorescence enhancing ligand and co-incubated with CTLs for $3 \mathrm{~h}$. T2 (-), untreated; T2 $(+)$, treated with MART1 A2 peptide, melanoma cell lines (C32: HLA-A*0201+, MART1+; RPMI7951: HLA-A*0201+, MART1*; NCC-KT: HLA-A*0201 $\left.{ }^{*}, \mathrm{MART}^{+}\right)$. Each column shows the mean $\pm \mathrm{SD}$ for triplicate samples.

\section{Results}

Tetramer $^{+}$CTL induction and expansion. After the expansion of melanoma peptide-specific CTLs, the frequency of MART-1 tetramer ${ }^{+}$CTLs increased to $46.5 \%$ (mean of 4 cases) compared with before stimulation $(<1 \%)$ (Table I). However, the expansion failed in 2 cases. Case 3 also demonstrated efficient expansion of gp100 A2 tetramer ${ }^{+}$CTLs (Table I, Fig. 1). In contrast, Flu-MP A2 tetramer ${ }^{+}$cells were not seen.

In Fig. 2, the absolute No. of MART-1 tetramer ${ }^{+}$CTLs was shown to increase from 187- to 619-fold (average 415-fold) after T2 stimulations compared to prior to the DC stimulation. In case 3 , gp100 A2 tetramer ${ }^{+}$CTLs were surprisingly expanded up to 1590 -fold (data not shown).
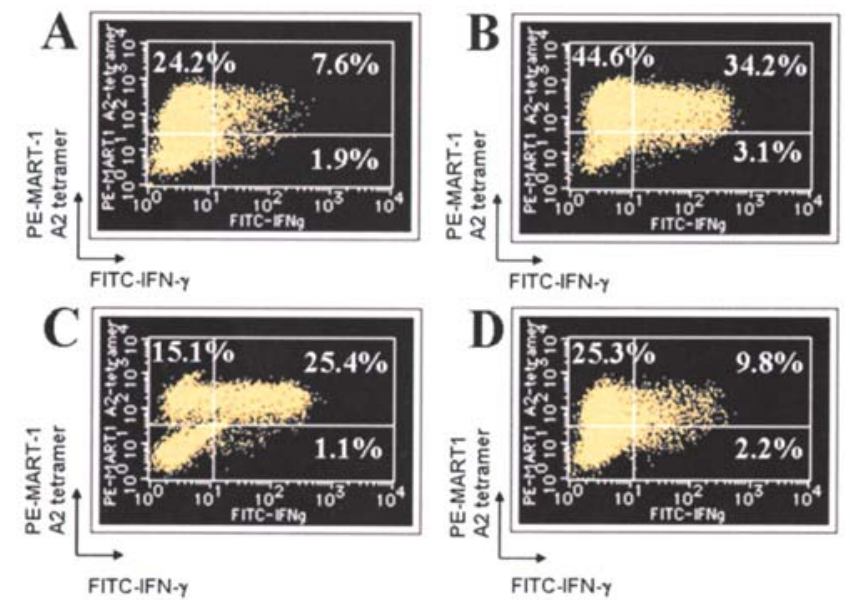

Figure 4. IFN- $\gamma$ production from MART-1 A2 peptide tetramer ${ }^{+}$CTL lines stimulated with peptide-pulsed T2 cells. Each CTL line was stained first with MART-1 A2 peptide tetramer and then intracellularly with anti-IFN- $\gamma$ MoAb. (A) case 1, (B) case 2, (C) case 3 and (D) case 4.

Table II. Frenquency of specific TCR repertoire ${ }^{+}$CTLs from HLA-A*0201 positive melanoma patients.

\begin{tabular}{llccc}
\hline $\begin{array}{l}\text { Case } \\
\text { no. }\end{array}$ & Peptide & $\begin{array}{c}\text { Tetramer }^{+} \\
\text {CTLs (\%) }\end{array}$ & Repertoire & $\begin{array}{c}\text { Frequency } \\
(\%)\end{array}$ \\
\hline 2 & MART-1 & 78.8 & BV9 & 35.7 \\
& & & BV20 & 18.5 \\
& & & BV4 & 12.1 \\
3 & MART-1 & 40.5 & BV27 & 11.3 \\
3 & gp100 & 44.3 & BV12 & 87.8 \\
\hline
\end{tabular}

${ }^{a}$ Frequency, percentage of tetramer ${ }^{+} /$specific TCR repertoir ${ }^{+}$CTLs. A TCR repertoire with a frequency of $>10 \%$ was chosen. Each value shows the mean for 2 experiments.
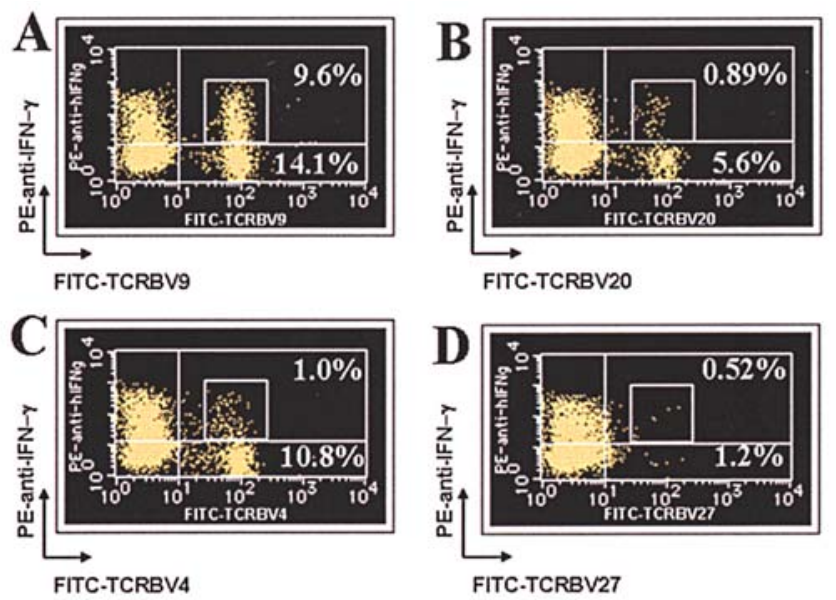

Figure 5 . IFN- $\gamma$ production by CTL populations recognized by specific antiTCR repertoire MoAb after stimulation with MART-1-pulsed T2 cells in case 2. (A) TCRBV9+, (B) TCRBV20+, (C) TCRBV4+ and (D) TCRBV27+ CTLs. Each CTL was stained first with anti-TCR repertoire MoAb and then intracellularly with anti-IFN- $\gamma$ MoAb after the T2 stimulation. 
A

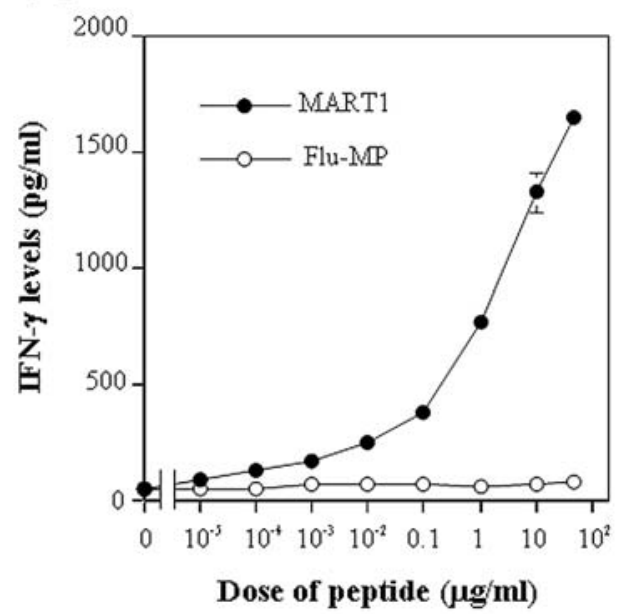

B

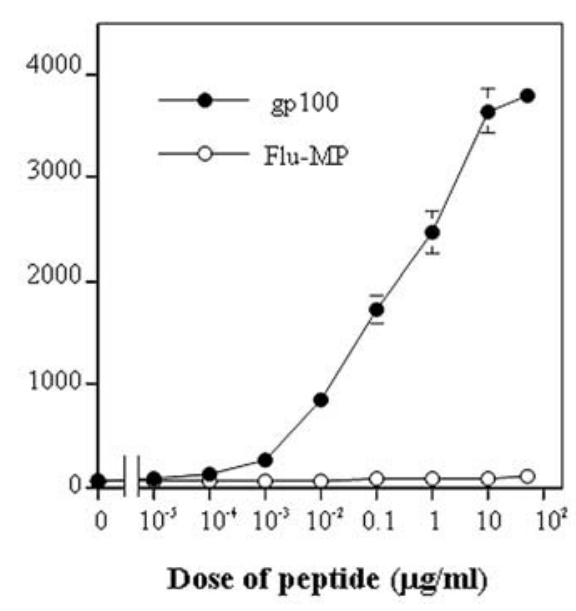

Figure 6. Peptide dose-dependent IFN- $\gamma$ productions from anti-TCR MoAb-sorted CTL lines. (A) anti-TCRBV28 MoAb-sorted MART-1-specific CTL line, (B) anti-TCRBV12 MoAb-sorted gp100-specific CTL line. These lines were stimulated with MART-1 or gp100-pulsed T2 cells. IFN- $\gamma$ levels in the supernatant were measured using human IFN- $\gamma$-specific ELISA kit. Each point shows the mean \pm SD of triplicate samples.

In contrast, the frequencies of MART-1 tetramer ${ }^{+}$CTLs from 4 patients prior to the DC vaccine were all $<5 \%$ even after ex vivo full expansion (data not shown). Additionally, the frequency of Flu-MP tetramer ${ }^{+}$(as a control) CTLs was $<0.2 \%$ after an expansion in all cases (data not shown).

CTL killing activity of expanded MART-1-specific CTLs. Cultured CTLs from 4 melanoma cases showed strong killing activity against MART-1 peptide-pulsed T2 cells and the C32 melanoma cell line (HLA-A*0201+, MART1 ${ }^{+}$) (Fig. 3). There was some difference in killing activity against $\mathrm{C} 32$ cells among cases. In contrast, no significant killing activity was seen in RPMI7951 (HLA-A*0201+, MART1') and NCC-KT (HLA-A*0201-, MART1+). The killing activity was shown to be HLA-A2 and antigen (MART-1)-specific.

Intracellular IFN- $\gamma$ staining of expanded CTLs from melanoma patients. The frequency of both MART-1 tetramer and IFN- $\gamma$-positive CTLs in 4 melanoma cases after peptidepulsed T2 stimulation was 7.6, 34.2, 25.4 and $9.8 \%$, respectively (Fig. 4). The percentage of IFN- $\gamma^{+}$out of all tetramer ${ }^{+}$cells was $23.9,43.4,62.7$ and $27.9 \%$. In either case CTLs from case 2 and 3 were more efficient in IFN- $\gamma$ production than those from the other two cases.

TCR repertoire profiling in melanoma cases and its relation to cytotoxic activity. After the expansion there were 1 major $(35.7 \%)$ and 3 minor $(18.5,12.1$ and $11.3 \%)$ populations with specific TCR repertoires among $78.8 \%$ of MART-1 tetramer ${ }^{+}$ CTLs in case 2 (Table II). Case 3 had a major population in both MART-1 (87.8\%) and gp100 (82.5\%) A2 tetramer ${ }^{+}$ CTLs.

Fig. 5 shows the association of IFN- $\gamma$ production by peptide-pulsed T2-stimulated CTLs (cytotoxic activity) with the specific TCR repertoire in case 2 . TCRBV9+ CTL populations alone exhibited a specific killing activity (TCRBV9+/IFN- $\gamma^{+} ; 9.6 \%$ ). Finally, TCRBV9+ CTLs in case 2 and $\mathrm{TCRBV} 28^{+}$or $\mathrm{BV} 12^{+}$in case 3 were specifically sorted (purity $>98 \%$ ) using the autoMACS system and all utilized for TCR gene cloning.

T2-stimulated IFN- $\gamma$ production from peptide-specific CTL line sorted by TCR-specific MoAb. CTL lines sorted by FITClabeled anti-TCRBV28 or anti-TCRBV12 MoAb showed MRAT-1 or gp100 A2-peptide specific cytotoxic activity, respectively (Fig. 6). Gp100 A2-peptide specific CTL clone exhibited greater IFN- $\gamma$ production than MART-1 peptidespecific clone after the various doses of peptide-pulsed T2 stimulation.

TCR cDNA sequences in MART-1 or gp100 A2 peptidespecific CTLs. Cloned TCR cDNA sequences are shown in Fig. 7 (MART-1-specific sequence in case 1, MART-1 or gp100-specific sequence in case 2). The TCR repertoire used was TCRBV9 in MART-1 A2 CTLs from case 2, and TCRBV28 and TCRBV12 in MART-1 and gp100 A2 CTLs from case 3 , respectively.

TCR cDNA transduction into primary naïve $T$ cells in melanoma cases. The GFP cDNA transduction experiment after antibody-mediated $\mathrm{T}$ cell stimulation showed that the activated state resulted in improved transduction efficiency (unstimulated $25.9 \%$ vs. stimulated $40.1 \%$ ) (Fig. 8). In the case of $4 \mu \mathrm{g}$ of the TCR cDNA for MART-1 and gp100, the frequency of TCR-positive T cells was 23.9\% (MART-1, case 1), 31.3\% (MART-1, case 2) and $13.3 \%$ (gp100, case 2), respectively (Fig. 9). cDNA ( $4 \mu \mathrm{g})$ and pre-activated T cells were more efficient in gene transduction than $2 \mu \mathrm{g}$ of cDNA and unactivated cells, respectively.

IFN- $\gamma$ production by TCR cDNA-transduced naïve T cells on antigen stimulation. PBMCs from melanoma patients were transduced with $4 \mu \mathrm{g}$ of TCR cDNA (MART-1 specific in case 2 and 3, gp100 specific in case 3) by electroporation and used for co-culture with peptide-pulsed T2 cells or the melanoma 


\section{Case2 MART-1 A2 CTL \\ Repertoire TRBV9*01 TRBD1*01 $\mathrm{P}$ N2 $\mathrm{P} \quad$ TRBJ2-7*01 \\ Nucleotide tgtgccagcagcgtag....gggc...g...gtc...ag....ctcetacgagcagtacttc \\ $\begin{array}{llllllllllllllllll}\text { Protein } & C & A & S & S & V & G & A & V & S & S & \mathbf{Y} & \mathbf{E} & \mathbf{Q} & \mathbf{Y} & \mathbf{F}\end{array}$}

\section{Case3 MART-1 A2 CTL}

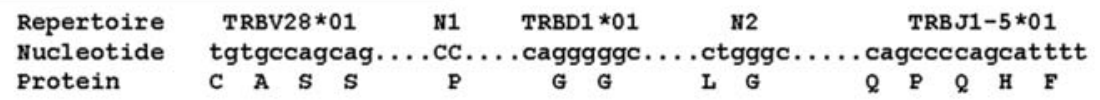

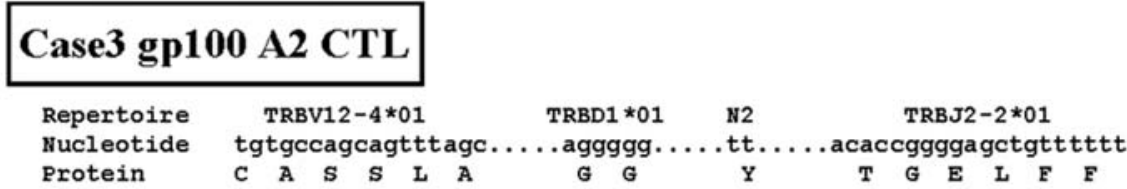

Firgure 7. Alignment of cloned TCR cDNA sequences from case 2 and 3. The TCR repertoire used was TCRBV9 in MART-1 A2 CTLs from case 2, and TCRBV28 and TCRBV12 in MART-1 and gp100 A2 CTLs from case 3, respectively. Segments V, D, J and C were identified using a tool at the IMTG web site (JunctionAnalysis, http://imgt.cines.fr/).

\section{A}

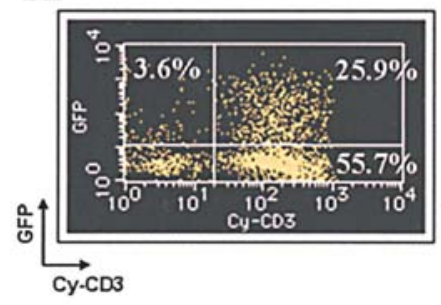

B

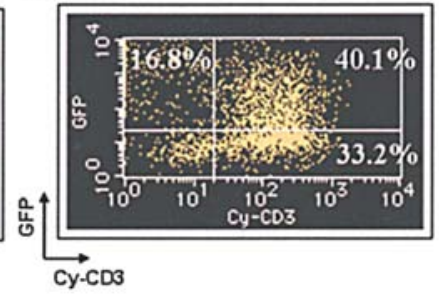

Figure 8. GFP gene transduction into PBMCs of melanoma patients using electroporation. After electroporation, cells were stained with anti-CD3 MoAb and analyzed on a flow cytometer. (A) unstimulated PBLs, (B) PBLs stimulated with anti-CD3 and anti-CD28 MoAb prior to electroporation.

cell line. PMBCs transduced with the MART-1-specific TCR cDNA (case 3) showed specific IFN- $\gamma$ production against MART-1 peptide-pulsed T2 cells and the HLA-A2 ${ }^{+}$, MART- $1^{+}$ C32 cell line in a HLA and antigen (MART-1)-restricted manner (Fig. 10). Additionally, PBMCs transduced with another MART-1-specific (case 2) or gp100-specific (case 3) TCR cDNA demonstrated specific IFN $-\gamma$ production against each of the peptide-pulsed T2 cells.

\section{Discussion}

It is accepted that spontaneously immunized CTL clones can be recognized at tumor sites or in peripheral blood without aggressive vaccinization, because melanomas are generally
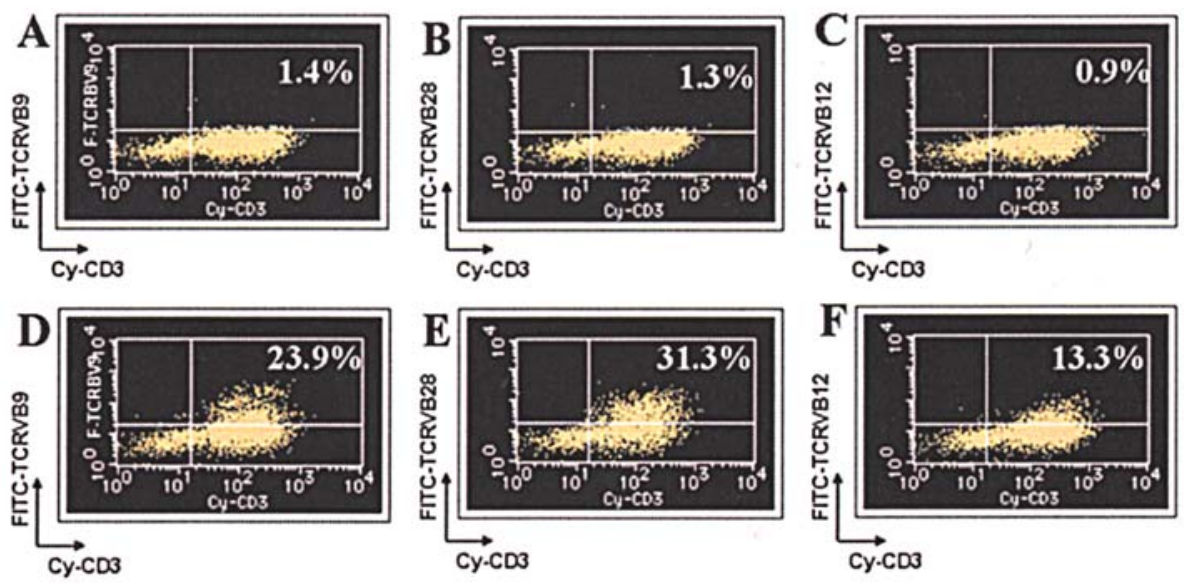

Figure 9. MART-1 or gp100 A2 peptide-specific TCR cDNA transduction into PBLs of HLA-A2 ${ }^{+}$melanoma patients. All PBMCs were stimulated with a cocktail of anti-CD3 and anti-CD28 MoAb prior to electroporation. Mock DNA was used in (A-C). Specific TCR DNA was transduced. (D) TCRBV9, (E) TCRBV28 and (F) TCRBV12. After electroporation, all cells were stained with specific anti-TCR repertoire MoAb and anti-CD3 antibody and analyzed on a flow cytometer. 
A

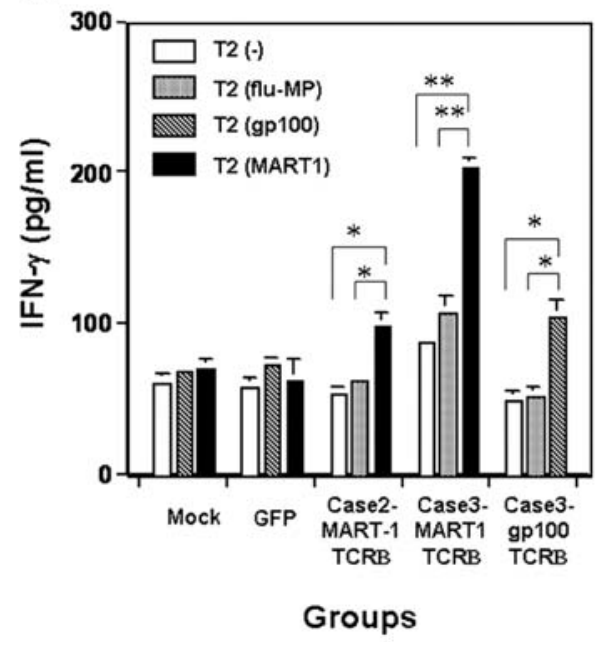

B

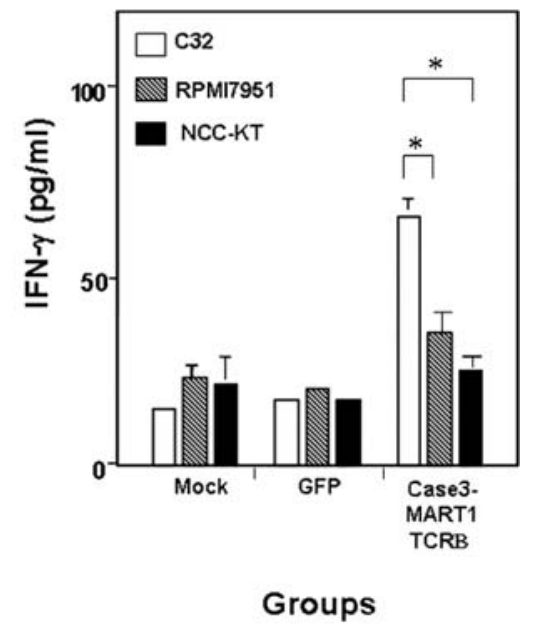

Figure 10. IFN- $\gamma$ production from specific TCR cDNA-transduced lymphocytes derived from a melanoma patient against melanoma cell lines. Mock or TCR gene-transduced lymphocytes were co-incubated with T2 cells (A) or a melanoma cell line (B) for $24 \mathrm{~h}$. The amount of IFN- $\gamma$ produced in the supernatant was measured using an ELISA kit. T2 (-), untreated; T2 (peptide), treated with various peptides, melanoma cell lines (C32, HLA-A*0201+, MART1+; RPMI7951, HLA-A*0201+, MART1 ${ }^{*}$; NCC-KT, HLA-A*0201-, MART1 ${ }^{+}$). Each column shows the mean \pm SD for triplicate samples. ${ }^{* *} \mathrm{P}<0.01,{ }^{*} \mathrm{P}<0.05$.

immunogenic tumors in terms of the immune response against antigens $(13,14)$. With regard to common melanoma antigens like MART-1, gp100 and tyrosinase, many heterogeneous tumor-infiltrating lymphocytes (TILs) or blood CTLs specific to these peptides have been identified using clonal analysis and characterized specifically in terms of antigen avidity and cytotoxic activity against tumors (15-18).

Previously, our group performed a peptide-cocktail pulsed DC-based immunotherapy in Japanese metastatic melanoma patients as a phase I/II study (4). This time we characterized melanoma antigen-specific CTL clones derived from the blood of patients given DC vaccines and established an ex vivo expansion culture method. Finally, our group succeeded in cloning and sequencing melanoma peptide (MART-1 and gp100)-specific T cell receptor (TCR) genes. Few clonal CTL analyses after the use of cancer vaccines including DCs and peptides have been performed so far (19-21). Powell et al (22) demonstrated the efficacy of a multiple course peptideimmunization strategy for the generation in high frequencies of tumor antigen-specific $\mathrm{T}$ cells, because they recognized circulating vaccine-specific CTLs in blood with an effector memory phenotype even one year after the final vaccination. Additionally, Godelaine et al (23) reported that several potent CTL clones specific to MAGE3 A1 peptide were amplified after the use of a peptide-pulsed DC vaccine and the frequency of tetramer-positive CTLs in blood increased 20-400-fold compared with before the vaccination.

As to ex vivo CTL expansion, we established our own method to increase number of melanoma-peptide-specific blood CTLs from patients given a DC vaccine several times. Briefly, PBMCs obtained from melanoma patients were stimulated twice in vitro with patient-derived DCs pulsed with the same peptide as used in the DC vaccine, and furthermore activated twice with peptide-pulsed T2 cells. Finally, MART-1 tetramer-positive CTLs were able to be expanded up to 415 -fold (on average) in 4 HLA-A2 ${ }^{+}$melanoma patients given the vaccine. These expanded CTLs were all efficient in killing MART-1 peptide ${ }^{+}$T2 or melanoma cells in a HLA and MART-1-restricted manner. This result was comparable to that obtained with MAGE3 A1 peptide reported by Godelaine et al. In contrast, in the case of CTLs obtained from patients prior to the vaccination, the expansion was much less extensive. This observation demonstrated that utilizing PBMCs from patients given a DC vaccine is a very efficient way of preparing numerous adoptive CTLs for clinical use. Different approaches to CTL expansion including the use of autologous DC (24), anti-CD3, CD28 antibody (equipped with beads) (25) or EB virus-transformed B cells (26) have been tried, however, it is considered that autologous DC with immunogenic peptides might be one of optimal expansion methods.

Additionally, distinguishing these CTLs in terms of tumorspecific avidity and cytotoxicity is important. Generally, tetramer-positive CTLs have polyclonal effectors and the clone responsible for the genuine anti-tumor activity cannot be identified at the expansion stage $(13,15,17)$. We utilized specific staining of CTLs with a combination of anti-TCR $\mathrm{MoAb}$ and intracellular IFN- $\gamma$ staining as shown in Fig. 5. Using this method, the monoclonal TCR repertoire mediating the anti-tumor cytotoxicity could be elucidated. Furthermore, anti-TCR MoAb-sorted CTL clones were shown to exhibit very potent melanoma-peptide specific cytotoxic activity (Fig. 6). Once the functional TCR repertoire is determined, a functional CTL clone can be purified by MoAb sorting, and finally specific DNA is cloned as we have performed. This might be a novel approach to determine the genuine clone responsible for peptide-specific cytotoxicity at the level of selection of polyclonal CTLs.

Evaluating the efficiency and capability of cancer-specific CTLs for clinical application is also an important issue. Many studies of cytotoxicity or avidity for tumors comparing TILs with blood CTLs have been performed. Basically, TIL clones tend to be more cytotoxic and have greater affinity for tumor cells and a more limited TCR repertoire than blood 
CTLs. Cole et al (27) and others showed that the same TCR repertoire specific to MART-1 peptide was recognized among blood CTLs as TIL clones isolated from tumors, and supported the application of vaccine-boosted blood CTLs to adoptive immunotherapy. Romero et al (28) have demonstrated that large numbers of tumor antigen-specific CTLs occurred in tumor-infiltrated lymph nodes, which were able to be expanded with cytokines efficiently. In the present study, TILs from melanoma tissue were not analyzed. In future, upcoming resected tumors will be used for TIL expansion according to the methods of others.

When considering the application of native adoptive CTL therapy, a great number of potent CTLs specific to cancer peptides are needed. The technology of TCR gene-engineering is possibly one efficient tool with which to expand the specific effector $\mathrm{T}$ cells. To date, retroviral vector-mediated TCR gene transduction has been utilized in basic research and some clinical trials (29-32). Recently, the use of a lentiviral vector system was reported to be the optimal way to transduce specific TCR genes into naïve T cells (33). However, adverse effects such as leukemogenesis in stem cell-based retroviral gene transduction programs cannot be avoided completely. In our study, a novel electroporation-based TCR gene transduction was performed and the transduction efficiency in naïve $\mathrm{T}$ cells derived from melanoma patients was acceptable (56\% for GFP gene, $31 \%$ for MART-1 TCRBV28 gene in case 3 , respectively). These rates are low compared with the optimal values for lentiviral-mediated transduction. However, CTLs after TCR gene transduction seemed to be functionally active and exhibited HLA-restricted cytotoxicity against not only $\mathrm{T} 2$ cells pulsed with peptides but also melanoma cell lines in a HLA-restricted manner. More importantly, antiCD3 and anti-CD28 antibody-mediated T cell activation prior to electroporation is needed to reduce the damage to $\mathrm{T}$ cells and promote the transduction efficiency as previously reported (34). The precise mechanism for the promoting effect of antibody stimulation on gene transduction remains to be elucidated. Our study demonstrated that the GFP and MART-1-specific TCRBV DNA transduction rate increased from 29.5 and $7.4 \%$ (without activation) to 56.9 and $31.3 \%$ (with activation), respectively (data not shown).

DC vaccine-based efficient CTL expansion using blood CTLs from vaccinated melanoma patients, may be a good immunotherapeutic modality. This novel approach can be employed for adoptive CTL therapy followed by the use of peptide-cocktail pulsed DC vaccines and the administration of a T cell-supporting cytokine such as IL-2, IL-7 or IL-15 to maintain and expand infused CTLs in vivo. As Dudley et al showed previously, positive approaches for immunomodulation such as lymphocyte depletion or the removal of regulatory $\mathrm{T}$ cells will be necessary to make a novel adoptive CTL therapy successful. Targeting immuno-suppressive molecules is another important approach that should be tried.

\section{Acknowledgements}

This work was supported in part by grant in cooperation of Innovative Technology and Advanced Research in Evolutional Area (City area) from the Ministry of Education, Culture,
Sports, Science and Technology. We thank Dr T. Mochizuki for supplying several synthetic peptides, and Ms. A. Suzuki, Mrs. K. Kuromi and Y. Kawaguchi for their excellent technical assistance.

\section{References}

1. Renkvist N, Castelli C, Robbins PF and Parmiani G: A listing of human tumor antigens recognized by $\mathrm{T}$ cells. Cancer Immunol Immunother 50: 3-15, 2001.

2. Nestle FO, Alijagic S, Gilliet M, et al: Vaccination of melanoma patients with peptide- or tumor lysate-pulsed dendritic cells. Nat Med 4: 328-332, 1998.

3. Banchereau J, Palucka AK, Dhodapkar M, et al: Immune and clinical responses in patients with metastatic melanoma to $\mathrm{CD} 34^{+}$progenitor-derived dendritic cell vaccine. Cancer Res 61: 6451-6458, 2001

4. Akiyama Y, Tanosaki R, Inoue N, et al: Clinical response in Japanese metastatic melanoma patients treated with peptide cocktail-pulsed dendritic cells. J Transl Med 3: 4, 2005.

5. Dudley ME, Wunderlich J, Nishimura MI, et al: Adoptive transfer of cloned melanoma-reactive $\mathrm{T}$ lymphocytes for the treatment of patients with metastatic melanoma. J Immunother 24: 363-373, 2001.

6. Yee C, Thompson JA, Byrd D, et al: Adoptive T cell therapy using antigen-specific $\mathrm{CD}^{+} \mathrm{T}$ cell clones for the treatment of patients with metastatic melanoma: in vivo persistence, migration, and antitumor effect of transferred T cells. Proc Natl Acad Sci USA 99: 16168-16173, 2002.

7. Dudley ME, Wunderlich JR, Robbins PF, et al: Cancer regression and autoimmunity in patients after clonal repopulation with antitumor lymphocytes. Science 298: 850-854, 2002.

8. Dudley ME, Wunderlich JR, Yang JC, et al: Adoptive cell transfer therapy following non-myeloablative but lymphodepleting chemotherapy for the treatment of patients with refractory metastatic melanoma. J Clin Oncol 23: 2346-2357, 2005.

9. Hoshino T, Seki N, Kikuchi M, et al: HLA class-I-restricted and tumor-specific CTL in tumor-infiltrating lymphocytes of patients with gastric cancer. Int J Cancer 70: 631-638, 1997.

10. Gohara R, Nakao M, Ogata Y, Isomoto H, Oizumi K and Itoh K: Histocompatibility leukocyte antigen-A2402-restricted cytotoxic $\mathrm{T}$ lymphocytes recognizing adenocarcinoma in tumor-infiltrating lymphocytes of patients with colon cancer. Jpn J Cancer Res 88: 198-204, 1997.

11. Akiyama Y, Maruyama K, Nara N, et al: Cytotoxic T cell induction against human malignant melanoma cells using HLA-A24-restricted melanoma peptide cocktail. Anticancer Res 24: 571-578, 2004.

12. Akiyama Y, Kuzushima K, Tsurumi $\mathrm{T}$ and Yamaguchi $\mathrm{K}$ : Analysis of HLA-A24-restricted CMVpp65 peptide-specific CTL with HLA-A*2402-CMVpp65 tetramer. Immunol Lett 95: 199-205, 2004.

13. Mandruzzato S, Rossi E, Bernardi F, et al: Large and dissimilar repertoire of Melan-A/MART-1-specific CTL in metastatic lesions and blood of a melanoma patient. J Immunol 169: 4017-4024, 2002.

14. Sensi M, Traversari C, Radrizzani M, et al: Cytotoxic T-lymphocyte clones from different patients display limited $\mathrm{T}$ cell-receptor variable-region gene usage in HLA-A2-restricted recognition of the melanoma antigen Melan-A/MART-1. Proc Natl Acad Sci USA 92: 5674-5678, 1995.

15. Valmori D, Dutoit V, Lienard D, et al: Tetramer-guided analysis of TCR B-chain usage reveals a large repertoire of Melan-Aspecific $\mathrm{CD}^{+} \mathrm{T}$ cells in melanoma patients. J Immunol 165 : 533-538, 2000.

16. Sensi M, Salvi S, Castelli, et al: T cell receptor (TCR) structure of autologous melanoma-reactive cytotoxic T lymphocyte (CTL) clone:tumor-infiltrating lymphocytes overexpress in vivo the TCR beta chain sequence uses by an HLA-A2-restricted and melanocyte-lineage-specific CTL clone. J Exp Med 178: 1231-1246, 1993.

17. Yee C, Savage PA, Lee PP, Davis MM and Greenberg PD: Isolation of high avidity melanoma-reactive CTL from heterogeneous populations using peptide-MHC tetramers. J Immunol 162: 2227-2234, 1999. 
18. Hishii M, Andrews D, Boyle LA, et al: In vivo accumulation of the same anti-melanoma T cell clone in two different metastatic sites. Proc Natl Acad Sci USA 94: 1378-1383, 1997.

19. Valmori D, Dutoit V, Schnuriger V, et al: Vaccination with a Melan-A peptide selects an oligoclonal T cell population with increased functional avidity and tumor reactivity. J Immunol 168: 4231-4240, 2002.

20. Kan-Mitchell J, Huang XQ, Steinman L, et al: Clonal analysis of in vivo activated $\mathrm{CD} 8^{+}$cytotoxic $\mathrm{T}$ lymphocytes from a melanoma patient responsive to active specific immunotherapy. Cancer Immunol Immunother 37: 15-25, 1993.

21. Powell DJ Jr, Dudley ME, Hogan KA, Wunderlich JR and Rosenberg SA: Adoptive transfer of vaccine-induced peripheral blood mononuclear cells to patients with metastatic melanoma following lymphodepletion. J Immunol 177: 6527-6539, 2006.

22. Powell DJ Jr and Rosenberg SA: Phenotypic and functional maturation of tumor antigen-reactive $\mathrm{CD} 8^{+} \mathrm{T}$ lymphocytes in patients undergoing multiple course peptide vaccination. J Immunother 27: 36-47, 2004.

23. Godelaine D, Carrasco J, Lucas S, et al: Polyclonal CTL responses observed in melanoma patients vaccinated with dendritic cells pulsed with a MAGE-3.A1 peptide. J Immunol 171: 4893-4897, 2003.

24. Kass R, Bellone S, Palmieri, et al: Restoration of tumor-specific HLA class I restricted cytotoxicity in tumor infiltrating lymphocytes of advanced breast cancer patients by in vitro stimulation with tumor antigen-pulsed autologous dendritic cells. Breast Cancer Res Treat 80: 275-285, 2003.

25. Ito F, Carr A, Svensson H, Yu J, Chang AE and Li Q: Antitumor reactivity of anti-CD3/anti-CD28 bead-activated lymphoid cells:implications for cell therapy in a murine model. J Immunother 26: 222-233, 2003.

26. Sili U, Huls MH, Davis AR, et al: Large-scale expansion of dendritic cell-primed polyclonal human cytotoxic T-lymphocyte lines using lymphoblastoid cell lines for adoptive immunotherapy. J Immunother 26: 241-256, 2003.
27. Cole DJ, Wilson MC, Rivoltini L, Custer M and Nishimura MI: T-cell receptor repertoire in matched MART-1 peptide-stimulated peripheral blood lymphocytes and tumor-infiltrating lymphocytes. Cancer Res 57: 5320-5327, 1997.

28. Romero P, Dunbar PR, Valmori D, et al: Ex vivo staining of metastatic lymph nodes by class I major histocompatibility complex tetramers reveals high numbers of antigen-experienced tumor-specific cytolytic T lymphocytes. J Exp Med 188: 1641-1650, 1998.

29. Roszkowski JJ, Lyons GE, Kast WM, Yee C, Van Besien K and Nishimura MI: Simultaneous generation of $\mathrm{CD}^{+}$and $\mathrm{CD} 4^{+}$ melanoma-reactive $\mathrm{T}$ cells by retroviral-mediated transfer of a single T-cell receptor. Cancer Res 65: 1570-1576, 2005.

30. Hughes MS, Yu YYL, Dudley ME, et al: Transfer of a TCR gene derived from a patient with a marked antitumor response conveys highly active $\mathrm{T}$ cell effector functions. Hum Gene Ther 16: 457-472, 2005.

31. Morgan RA, Dudley ME, Wunderlich JR, et al: Cancer regression in patients after transfer of genetically engineered lymphocytes. Science 314: 126-129, 2006.

32. Tsuji T, Yasukawa M, Matsuzaki J, et al: Generation of tumorspecific, HLA class I-restricted human Th1 and Tc1 cells by cell engineering with tumor peptide-specific T-cell receptor genes. Blood 106: 470-476, 2005.

33. Van Tendeloo V, Willems R, Ponsaerts P, et al: High-level transgene expression in primary human $\mathrm{T}$ lymphocytes and adult bone marrow $\mathrm{CD} 34^{+}$cells via electroporation-mediated gene delivery. Gene Ther 7: 1431-1437, 2007.

34. Chun HJ, Zheng L, Ahmad M, et al: Pleiotropic defects in lymphocytes activation caused by caspase- 8 mutations lead to human immunodeficiency. Nature 419: 395-399, 2002. 